\title{
Analytic and numerical study of a hierarchical spin model
}

\author{
Yu.Kozitsky ${ }^{1,2}$, M.Kozlovskii ${ }^{2}$, T.Krokhmalskii ${ }^{2}$ \\ 1 Institute of Mathematics of Marie Curie-Sklodowska University, \\ 20-031 Lublin, Poland \\ 2 Institute for Condensed Matter Physics \\ of the National Academy of Sciences of Ukraine, \\ 1 Svientsitskii Str., 290011 Lviv, Ukraine
}

Received October 17, 1997

A simple hierarchical scalar spin model is studied analytically and numerically in the vicinity of its critical point. The dependence of the finite size (i.e. calculated for a large but finite number of spins) susceptibility and the location of zeros of the model partition function on the number of spins at the critical point is described analytically. It is also shown analytically that the finite size correlation length in such a model diverges at the critical point slower than it is supposed in the finite size scaling theory. Certain numerical information about the critical point and ordered phase is given. In particular, the critical temperature of the model and the critical index describing the order parameter are calculated for various values of the interaction parameter.

Key words: hierarchical models, Ising-like spin models, one-dimensional systems, critical point, phase transition

PACS: $05.50 .+q, 64.60 . \mathrm{Cn}, 64.60 . \mathrm{Fr}, 75.10 . \mathrm{Hr}$

\section{Introduction}

Hierarchical models play a significant role in modern classical statistical physics. First such model was introduced by F.Dyson [6] in 1969 as a tool in the study of one-dimensional Ising-like spin models with long-range interaction potentials. Since that time these models have been studied and utilized. An excellent survey of ideas and results in this domain can be found in [3].

The main idea of F.Dyson was to substitute a one-dimensional Ising-like spin model with the translation invariant interaction potential which decays as a power of the spin-spin distance by a model with the interaction decaying as the same power, but of the distance defined by special non-Euclidean metric and possessing some other symmetry instead of the translation invariance. This new symmetry 
was designed to allow a more detailed and mathematically strict description which would also yield some information about the properties of the initial translation invariant model.

On the other hand, in the 70-ies I.Yukhnovskii and his collaborators developed an approach to the study of a three-dimensional Ising model based on the collective variables method (see, e.g., [21], [22]). The main tool of this approach was a step-by-step integration scheme which allowed one to obtain the model partition function, free energy and other thermodynamic functions in the vicinity of the critical point [17]. The peculiarity of this scheme lies in the fact that the partition function is calculated as a product of the partial partition functions describing a sequence of growing boxes of spins. It was considered as a realization of the known heuristic Kadanoff block-spin construction which was intended to describe the critical points of such spin models. This construction was based on the idea that the ensembles of properly renormalized total spins of cubic blocks of various linear sizes are distributed identically whenever the model reaches its critical point. Such property is known as a critical point self-similarity [20] or as a critical point scale invariance. The latter is considered to be the main symmetry appearing at the critical point.

In fact, the level of strictness of the Yukhnovskii approach is rather physical, which means that certain approximations with no quantitatively controlled consequences were employed. Later it was understood [13] that the qualitative result of these approximations lay in the replacement of the translation invariance of the model considered by the self-similarity symmetry which was embodied in the Kadanoff construction. Moreover, it was shown in paper [13] (see also [14] and [10]) that the step-by-step integration scheme, being applied to a translation invariant spin model, transforms it into a model identical to the Dyson's hierarchical model. In what follows, one can consider the Dyson model as a realization of the Yukhnovskii scheme of the study of translation invariant Ising-like spin models.

Since early 70-ies, the Dyson hierarchical model was studied mainly analytically. At the same time investigations of a three-dimensional Ising model within the collective variables method by I.Yukhnovskii and his collaborators (including numerical ones) were carried out. As a result, universal and nonuniversal aspects of the specific heat behaviour in the vicinity of the critical point were analyzed $[22],[15]$. An equation of state was derived, and its analytic solution describing the order parameter dependence on temperature, external magnetic field and microscopic model parameters in the vicinity of the critical point was found [5], [16]. It should be noted that a number of additional approximations were used to obtain these results.

The aim of our work is to study - analytically and numerically - a simple hierarchical model, considering it also as an emanation from the Yukhnovskii scheme, in the vicinity of its critical point without the additional approximations just mentioned. The main question here is how the quantities which describe the behaviour of the model in the vicinity of the critical point (such as susceptibility, order parameter, correlation length, location of zeros of the partition function, etc.), calculated 
for the fixed large but finite number of spins, depend on thermodynamic variables, or how they depend on the number of spins if the thermodynamic variables are fixed at their critical values. Our analytic study is conducted by means of rigorous methods. We discuss the asymptotic behaviour of the mentioned quantities, calculated at the critical point, when the number of spins tends to infinity. For this aim the facts already known are taken from the corresponding papers, whereas a number of new ones are proven here. As a result, we have suggested how to calculate numerically the order parameter for zero values of the external magnetic field, how to find the corresponding critical index, how to calculate the critical temperature. Such an approach is partially inspired by works on the finite size scaling, [8] and [9] in particular. We suppose to use the finite size scaling methods for the description of our model on the basis of the data obtained here - it will be done in our next work. To prepare it we check the basic assumption of this method concerning the critical point correlation length asymptotics, with the size of the model tending to infinity, and find that it should be changed in the case of our model.

As tools we use the methods developed earlier [14], [10], [11], as well as direct numerical calculations of the corresponding quantities for a sufficiently large number of spins based on the analysis mentioned above. Such calculations became possible also due to a special (hierarchical) structure of the model considered.

\section{The model}

Here we give descriptions of the models and those facts from their theory which are related to our investigation. Some of these facts are proven here, others are taken from the papers published earlier.

Let us consider a countable set of one-dimensional spins $\left\{\sigma_{s} \in \mathbf{R}, s \in \mathbf{N}\right\}$ which we choose to be indexed simply by positive integers. The formal Hamiltonians of the translation invariant and hierarchical models are

$$
\begin{aligned}
H^{\mathrm{tr}} & =-\frac{1}{2} \sum_{s, s^{\prime} \in \mathbf{N}} J_{s s^{\prime}}^{\mathrm{tr}} \sigma_{s} \sigma_{s^{\prime}}-h \sum_{s \in \mathbf{N}} \sigma_{s} ; \\
H & =-\frac{1}{2} \sum_{s, s^{\prime} \in \mathbf{N}} J_{s s^{\prime}} \sigma_{s} \sigma_{s^{\prime}}-h \sum_{s \in \mathbf{N}} \sigma_{s} ;
\end{aligned}
$$

where $h$ is an external magnetic field, and

$$
J_{s s^{\prime}}^{\mathrm{tr}}=-J\left(\left|s-s^{\prime}\right|+1\right)^{-1-\lambda}, \quad J>0 .
$$

Here $|a|$ stands for the absolute value of $a \in \mathbf{Z}$. The function $\left|s-s^{\prime}\right|$ can be considered as the Euclidean distance on $\mathbf{N}$, it is invariant with respect to the shifts (translations) along $\mathbf{N}$. The parameter $\lambda$ describes a decay of the potential $J_{s s^{\prime}}^{\mathrm{tr}}$, it is a priori set to be positive. As to the potential $J_{s s^{\prime}}$ in the Hamiltonian (2.2), it is put to be of the form (2.3) but with the "hierarchical distance" between $s$ and $s^{\prime}$ instead 
of $\left|s-s^{\prime}\right|$. This new distance can be set by means of the hierarchical structure on $\mathbf{N}$. The latter is the sequence $\mathcal{L}=\left\{\mathcal{L}_{n}, n \in \mathbf{Z}_{+}\right\}$of families $\mathcal{L}_{n}=\left\{\Lambda_{n, r}, r \in \mathbf{N}\right\}$, where

$$
\Lambda_{n, r}=\left\{s \in \mathbf{N} \mid 2^{n}(r-1)+1 \leqslant s \leqslant 2^{n} r\right\} .
$$

These subsets of $\mathbf{N}$ obey the following recursive rule

$$
\Lambda_{n, r}=\bigcup_{s \in \Lambda_{1, r}} \Lambda_{n-1, s}
$$

Definition 2.1 Let $n, s, s^{\prime}$ be chosen on $\mathbf{N}$. The points $s$ and $s^{\prime}$ are said to be separated on the hierarchy level $n$ if they belong to different subsets $\Lambda$ from $\mathcal{L}_{n}$.

Proposition 2.1 For an arbitrary noncoinciding pair of points $s, s^{\prime} \in \mathbf{N}$, there exits $n \in \mathbf{N}$ such that these points are separated on the hierarchy level $n-1$ and are not separated on the levels $n, n+1, \ldots$

This number is denoted as $n\left(s, s^{\prime}\right)$, that is

$$
n\left(s, s^{\prime}\right)=\min \left\{n \in \mathbf{N} \mid\left(\exists \Lambda \in \mathcal{L}_{n}\right)(s \in \Lambda) \&\left(s^{\prime} \in \Lambda\right)\right\}
$$

For example, $n(1,2)=1 ; n(2,3)=2 ; n(4,5)=3$. By means of $n\left(s, s^{\prime}\right)$, we can define the hierarchical distance on $\mathbf{N}$ :

$$
\operatorname{dist}\left(s, s^{\prime}\right)=2^{n\left(s, s^{\prime}\right)}-1 .
$$

Then the interaction potential in the Hamiltonian (2.2) is written as follows:

$$
J_{s s^{\prime}}=J\left(\operatorname{dist}\left(s, s^{\prime}\right)+1\right)^{-1-\lambda},
$$

where $\lambda$ and $J$ are the same as in (2.3). In order to relate such a model to the $d$-dimensional translation invariant model considered within the Yukhnovskii scheme, one should put $\lambda=2 / d$ (see [13], [14], [10]).

The following assertion can be proven directly from the definition.

Proposition 2.2 For an arbitrary pair of points $s, s^{\prime} \in \mathbf{N}$,

$$
\left|s-s^{\prime}\right| \leqslant \operatorname{dist}\left(s, s^{\prime}\right)
$$

that yields

$$
J_{s s^{\prime}}^{\mathrm{tr}} \geqslant J_{s s^{\prime}}
$$

Thermodynamic properties of the model can be described by passing to the limit $\Lambda \nearrow \mathbf{N}$ for the expectations $\left\langle. .>_{\Lambda}\right.$ computed at the finite subset $\Lambda$ with the help of Gibbs measures $\mu_{\Lambda}^{\beta, h}$. They are defined as probability measures on the configuration spaces $\mathbf{R}^{\Lambda}$ by means of local Hamiltonians $H_{\Lambda}\left(\sigma_{\Lambda}\right)$, where $\sigma_{\Lambda}=$ $\left\{\sigma_{s}, s \in \Lambda\right\}$. The local Hamiltonians are constructed according to the formal ones 
(2.1), (2.2) described just below. Here we consider only the Gibbs measures which correspond to zero boundary conditions. Thus,

$$
\delta \mu_{\Lambda}^{\beta, h}\left(\sigma_{\Lambda}\right)=\frac{1}{Z_{\Lambda}^{\beta, h}} \exp \left(-\beta H_{\Lambda}\left(\sigma_{\Lambda}\right)\right) \prod_{s \in \Lambda} \delta \chi\left(\sigma_{s}\right)
$$

where $Z_{\Lambda}^{\beta, h}$ is a normalizing constant which provides for $\mu_{\Lambda}^{\beta, h}$ to be probabilistic, and the measure $\chi$ describes the single-spin (even) probability distribution. The simplest case of the latter is the measure concentrated at points \pm 1 that corresponds to the Ising-like spins.

For hierarchical models, the thermodynamic limit is naturally achieved within the hierarchical structure, that is by choosing $\Lambda \in \mathcal{L}_{n}$ and putting $n \rightarrow \infty$. Hence, we may set the hierarchical local Hamiltonians only for such $\Lambda$. Thus, we use relation (2.5) and define the family of local Hamiltonians $\left\{H_{\Lambda_{n, r}} \mid n \in \mathbf{Z}_{+}, r \in \mathbf{N}\right\}$ recursively by putting

$$
H_{\Lambda_{n, r}}\left(\sigma_{\Lambda_{n, r}}\right)=-\frac{1}{2} 2^{-n(1+\lambda)}\left(1-2^{-1-\lambda}\right) J \sigma^{2}\left(\Lambda_{n, r}\right)+\sum_{s \in \Lambda_{1, r}} H_{\Lambda_{n-1, s}}\left(\sigma_{\Lambda_{n-1, s}}\right)
$$

with

$$
H_{\Lambda_{0, s}}\left(\sigma_{s}\right)=-\frac{1}{2}\left(1-2^{-1-\lambda}\right) J \sigma_{s}^{2}-h \sigma_{s}
$$

where

$$
\sigma(\Lambda)=\sum_{s \in \Lambda} \sigma_{s}
$$

Clearly, these Hamiltonians are invariant with respect to those permutations of $\mathbf{N}$ which preserve the hierarchical structure. Such permutations form a group which is the symmetry group of the model. In particular, all Hamiltonians with the same $n$ and different $r \in \mathbf{N}$ are identical, therefore, can be represented by one of them, $H_{\Lambda_{n, 1}}$ for example. The relationship between the Hamiltonians (2.2) and (2.12) can be established as follows. We rewrite $H_{\Lambda_{n, 1}}$ given by $(2.12)$

$$
H_{\Lambda_{n, 1}}\left(\sigma_{\Lambda_{n, 1}}\right)=-\frac{1}{2} \sum_{s, s^{\prime} \in \Lambda_{n, 1}} J_{s s^{\prime}}^{(n)} \sigma_{s} \sigma_{s^{\prime}}-h \sum_{s \in \Lambda_{n, 1}} \sigma_{s}
$$

that can be used as a definition of $J_{s s^{\prime}}^{(n)}$. Having in mind that $\Lambda_{n, 1}$ absorbs $\mathbf{N}$ when $n \rightarrow \infty$ and using relations (2.12)-(2.14), one can prove such a statement.

\section{Proposition 2.3}

$$
\lim _{n \rightarrow \infty} J_{s s^{\prime}}^{(n)}=2^{-n\left(s, s^{\prime}\right)(1+\lambda)} J .
$$

Along with the Hamiltonians (2.15) we will also consider the local Hamiltonian defined by the Hamiltonian (2.1)

$$
H_{\Lambda_{n, r}}^{\mathrm{tr}}(\sigma)=-\frac{1}{2} \sum_{s, s^{\prime} \in \Lambda_{n, r}} J_{s s^{\prime}}^{\mathrm{tr}} \sigma_{s} \sigma_{s^{\prime}}-h \sum_{s \in \Lambda_{n, r}} \sigma_{s}
$$


where $J_{s s^{\prime}}^{\mathrm{tr}}$ is given by $(2.3)$.

Consider the Gibbs measures (2.11) of the hierarchical model with $\Lambda=\Lambda_{n, r}$. Relation (2.12) yields the following recursion formula:

$$
\begin{aligned}
\mathrm{d} \mu_{\Lambda_{n, r}}^{\beta, h}\left(\sigma_{\Lambda_{n, r}}\right)= & Y_{n}^{\beta, h} \exp \left\{\frac{\beta}{2}\left(1-2^{-1-\lambda}\right) 2^{-n(1+\lambda)} J \sigma^{2}\left(\Lambda_{n, r}\right)\right\} \\
& \times \prod_{s \in \Lambda_{1, r}} \mathrm{~d} \mu_{\Lambda_{n-1, s}}^{\beta, h}\left(\sigma_{\Lambda_{n-1, s}}\right),
\end{aligned}
$$

where

$$
Y_{n}^{\beta, h}=\left(Z_{\Lambda_{n-1,1}^{\beta, h}}^{\beta}\right)^{2} / Z_{\Lambda_{n, 1}}^{\beta, h}
$$

Here we have taken into account that $Z_{\Lambda_{n, r}}^{\beta, h}=Z_{\Lambda_{n, 1}}^{\beta, h}$ for all $r \in \mathbf{Z}$. Due to the hierarchical symmetry of the model all sums $\sigma\left(\Lambda_{n, r}\right)$ with the same $n$ and different $r$ are distributed identically. Denote

$$
P_{\Lambda_{n, r}}^{\beta, h}(A)=\operatorname{Prob}\left(\sigma\left(\Lambda_{n, r}\right) \in A\right)
$$

for every Borel subset $A \subset \mathbf{R}$. Then

$$
\frac{\delta P_{\Lambda_{n, r}}^{\beta, h}(\xi)}{d \xi}=\int_{\mathbf{R}^{\Lambda_{n, r}}} \delta\left(\sigma\left(\Lambda_{n, r}\right)-\xi\right) \delta \mu_{\Lambda_{n, r}, h}^{\beta, h}\left(\sigma_{\Lambda_{n, r}}\right),
$$

where $\delta(\ldots)$ is the Dirac $\delta$ - function. Thus, relation (2.17) yields for such $P_{\Lambda_{n, r}, h}^{\beta}$ :

$$
\begin{aligned}
\mathrm{d} P_{\Lambda_{n, r}}^{\beta, h}(\xi) & =Y_{\Lambda_{n, 1}}^{\beta, h} \exp \left\{\frac{\beta}{2} 2^{-n(1+\lambda)}\left(1-2^{-1-\lambda}\right) J \xi^{2}\right\} Q_{\Lambda_{n, r}}^{\beta, h}(\xi) \delta \xi \\
Q_{\Lambda_{n, r}}^{\beta, h}(\xi) & =\int_{\mathbf{R}} \delta\left(\sum_{s \in \Lambda_{1, r}} \xi_{s}-\xi\right) \prod_{s \in \Lambda_{1, r}} \mathrm{~d} P_{\Lambda_{n-1, s}, h}^{\beta, h}\left(\xi_{s}\right) .
\end{aligned}
$$

In the case where the initial measure $P_{\Lambda_{0, s}}^{\beta, h}$ is absolutely continuous with respect to the Lebesgue measure on $\mathbf{R}$, all $P_{\Lambda_{n, r}}^{\beta, h}$ possess this property. It means that the tempered distribution introduced by (2.18) now is a suitable function. We denote it as

$$
\frac{\mathrm{d} P_{\Lambda_{n, r}}^{\beta, h}(\xi)}{\mathrm{d} \xi}=T_{n}^{\beta, h}(\xi),
$$

and remark that the so-called "small" partition function $F_{n}^{\beta, h}(x)$ studied in papers [1], [2] is related to our $T_{n}^{\beta, h}$ as follows:

$$
\begin{aligned}
F_{n}^{\beta, h}(x) & \left.:=\int_{\Lambda_{n, 1}} \delta\left(2^{-n} \sigma\left(\Lambda_{n, 1}\right)-x\right) \delta \mu_{\Lambda_{n, 1}, h}^{\beta, \sigma_{\Lambda_{n, 1}}}\right) \\
& =T_{n}^{\beta, h}\left(2^{n} x\right), \quad x \in \mathbf{R} .
\end{aligned}
$$


The recursive relation given by (2.19), (2.20) now takes the form

$$
\begin{aligned}
T_{n}^{\beta, h}(\xi)= & \frac{1}{K_{n}^{\beta, h}} \exp \left(\frac{\beta}{2} 2^{-n(1+\lambda)}\left(1-2^{-1-\lambda}\right) J \xi^{2}\right) \\
& \times \int_{\mathbf{R}} T_{n-1}^{\beta, h}\left(\frac{\xi}{2}+\eta\right) T_{n-1}^{\beta, h}\left(\frac{\xi}{2}-\eta\right) \mathrm{d} \eta,
\end{aligned}
$$

where

$$
\begin{aligned}
K_{n}^{\beta, h}= & \int_{\mathbf{R}^{2}} \exp \left(\frac{\beta}{2} 2^{-n(1+\lambda)}\left(1-2^{-1-\lambda}\right) J \xi^{2}\right) \\
& \times T_{n-1}^{\beta, h}\left(\frac{\xi}{2}+\eta\right) T_{n-1}^{\beta, h}\left(\frac{\xi}{2}-\eta\right) \mathrm{d} \xi d \eta
\end{aligned}
$$

Here we have taken into account that all $\Lambda_{1, r}$ consist of two points, and all $\sigma\left(\Lambda_{n, r}\right)$ with the same $n$ and different $r$ are distributed identically.

Now let us describe in detail the family of measures which are to be chosen in this research as initial single-spin measures $\chi$. The first condition imposed on these measures is the existence of their Laplace transforms

$$
f_{\chi}(z)=\int_{\mathbf{R}} \exp (z \sigma) \mathrm{d} \chi(\sigma), \quad z \in \mathbf{C}
$$

as entire functions. Let $\mathcal{F}$ be a family of entire functions of one complex variable possessing the following canonical representation:

$$
\begin{aligned}
& f(z)=\exp \left(\theta z^{2}\right) \prod_{j=1}^{\infty}\left(1+\gamma_{j} z^{2}\right) \\
& \theta \geqslant 0, \quad \gamma_{j} \geqslant 0, \quad \sum_{j=1}^{\infty} \gamma_{j}<\infty, \quad \gamma_{j} \geqslant \gamma_{j+1} .
\end{aligned}
$$

In other words, the family $\mathcal{F}$ consists of entire functions with the order of growth two at most which either have purely imaginary zeros or have them none.

Definition 2.2 A probability measure $\chi$ is said to possess the Lee-Yang property if its Laplace transform (2.25) belongs to the family $\mathcal{F}$.

It can be shown that the measures

$$
\delta \chi(\sigma)=\frac{1}{2}\{\delta(\sigma-1)+\delta(\sigma+1)\} \delta \sigma
$$

and

$$
\mathrm{d} \chi(\sigma)=\frac{\exp \left(-\frac{1}{2} u \sigma^{2}-\frac{1}{4} v \sigma^{4}\right) \mathrm{d} \sigma}{\int_{\mathbf{R}} \exp \left(-\frac{1}{2} u \sigma^{2}-\frac{1}{4} v \sigma^{4}\right) \mathrm{d} \sigma}, u \in \mathbf{R}, v>0,
$$

possess the Lee-Yang property. Further details can be found in paper [12]. Here we discuss only the properties of those measures which are relevant to our research. 
As can be seen from definition (2.25), the moments of each such measure can be computed as corresponding derivatives at zero of its Laplace transform $f_{\chi}$. The following derivatives

$$
u_{\chi}^{(2 k)}=\left(D^{2 k} \log f_{\chi}\right)(0), k \in \mathbf{N}, D=\frac{\mathrm{d}}{\mathrm{d} z},
$$

are known as semiinvariants or cumulants of $\chi$. Directly from definition (2.26) one obtains the following representations for these parameters:

$$
\begin{aligned}
u_{\chi}^{(2)} & =2\left(\theta+\Gamma_{1}\right), \\
u_{\chi}^{(2 k)} & =(-1)^{k-1} 2(2 k-1) ! \Gamma_{k}, \quad k \geqslant 2, \\
\Gamma_{k} & =\sum_{j=1}^{\infty} \gamma_{j}^{k}, \quad k \in \mathbf{N} .
\end{aligned}
$$

The only measure possessing the Lee-Yang property, the Laplace transform of which has no zeros, is the Gaussian measure. All the others can be characterized by the location of its zeros which are nearest to the origin of the imaginary axis. These are at points $\pm z_{1}= \pm \mathrm{i} \gamma_{1}^{-1 / 2}$ (see (2.26)).

Proposition 2.4 Let for a non-Gaussian measure $\chi$ possessing the Lee-Yang property, the semiinvariants be defined by (2.29). Then the location of the nearest zeros of its Laplace transform obeys the following two-sided estimate:

$$
\left[40 \frac{\left|u_{\chi}^{(4)}\right|}{u_{\chi}^{(2)} u_{\chi}^{(6)}}\right]^{\frac{1}{4}} \leqslant\left|z_{1}\right| \leqslant\left[6 \frac{u_{\chi}^{(2)}}{\left|u_{\chi}^{(4)}\right|}\right]^{\frac{1}{2}} .
$$

The proof of this statement can be done by showing the validity of such two-sided estimate for $\gamma_{1}$ :

$$
\frac{\Gamma_{2}}{\theta+\Gamma_{1}} \leqslant \gamma_{1} \leqslant \sqrt{\frac{\left(\theta+\Gamma_{1}\right) \Gamma_{3}}{\Gamma_{2}}},
$$

which in its turn immediately implies (2.31). To prove (2.32) one can use the following form of the Cauchy inequality:

$$
\Gamma_{2}^{2}=\left[\sum_{j=1}^{\infty} \gamma_{j}^{1 / 2} \gamma_{j}^{3 / 2}\right]^{2} \leqslant\left[\sum_{j=1}^{\infty} \gamma_{j}\right]\left[\sum_{j=1}^{\infty} \gamma_{j}^{3}\right],
$$

which yields $\Gamma_{2}^{2} \leqslant \Gamma_{1} \Gamma_{3} \leqslant\left(\theta+\Gamma_{1}\right) \Gamma_{3}$. The latter estimate, together with the obvious relation $\gamma_{1}^{2} \leqslant \Gamma_{2}$, gives the upper bound for $\gamma_{1}$ in (2.32). The lower bound is the simple consequence of the following estimate $\Gamma_{2} \leqslant \gamma_{1} \Gamma_{1} \leqslant \gamma_{1}\left(\theta+\Gamma_{1}\right)$.

For the measure $\chi$, we set

$$
a_{\chi}=-u_{\chi}^{(4)} /\left(u_{\chi}^{(2)}\right)^{2}
$$

By means of the methods of paper [12] one can prove 
Proposition 2.5 Let the measure $\chi$ possess the Lee-Yang property. Then

$$
0 \leqslant a_{\chi} \leqslant 2
$$

Moreover, $a_{\chi}=0$ if, and only if, $\chi$ is Gaussian; $a_{\chi}=2$ if, and only if, $\chi$ is concentrated at points $\pm a$, i.e. is of the type (2.27) with some a $>0$ instead of 1 .

The following statement is a base for the application of the Lee-Yang property in the theory of hierarchical models. It was proven in [10] and applied in [11].

Proposition 2.6 Let the initial single-spin measure $\chi$ possess the Lee-Yang property. Then, all $P_{\Lambda_{n, r}, 0}^{\beta}$ defined by (2.11), (2.18), (2.19), (2.20) possess this property provided they exist as measures.

It was shown [11] that all $P_{\Lambda_{n, r}}^{\beta, h}$ exist as measures for all the values of $\beta$ if the initial measure $\chi$ is such that $f_{\chi}$ possesses the representation (2.26) with $\theta=0$. Only such initial measures are considered below, the measures (2.27), (2.28) obey this restriction.

\section{The critical point and an ordered phase}

We start with the description of an ordered phase. For this purpose we will need an order parameter. In spin models it should be magnetization per spin in a zero external field. It can be computed by means of the corresponding Gibbs measures. Consider the following expectations:

$$
\begin{gathered}
M_{n}^{(l)}(\beta, h)=<\left(\sigma\left(\Lambda_{n, 1}\right)\right)^{l}>_{\Lambda_{n, 1}} \\
=\int_{\mathbf{R}^{\Lambda_{n, 1}}}\left(\sigma\left(\Lambda_{n, 1}\right)\right)^{l} \mathrm{~d} \mu_{\Lambda_{n, 1}}^{\beta, h}\left(\sigma_{\Lambda_{n, 1}}\right) \\
=\int_{\mathbf{R}} \xi^{l} \mathrm{~d} P_{\Lambda_{n, 1}, h}^{\beta,}(\xi), l \in \mathbf{N} ; \\
\bar{M}_{n}(\beta)=\int_{\mathbf{R}^{\Lambda_{n, 1}}}\left|\sigma\left(\Lambda_{n, 1}\right)\right| \mathrm{d} \mu_{\Lambda_{n, 1}^{\beta, 0}}^{\beta}\left(\sigma_{\Lambda_{n, 1}}\right)=\int_{\mathbf{R}}|\xi| \mathrm{d} P_{\Lambda_{n, 1}}^{\beta, 0}(\xi), \\
\bar{m}_{n}(\beta)=\int_{\mathbf{R}^{\Lambda_{n, 1}}}\left|\sigma_{1}\right| \mathrm{d} \mu_{\Lambda_{n, 1}^{\beta, 0}}^{\beta}\left(\sigma_{\Lambda_{n, 1}}\right) ; \\
\tilde{m}_{n}^{(2)}(\beta)=\min _{r, s \in \Lambda_{n, 1}} \int_{\mathbf{R}^{\Lambda_{n, 1}}} \sigma_{r} \sigma_{s} \mathrm{~d} \mu_{\Lambda_{n, 1}, 0}^{\beta,}\left(\sigma_{\Lambda_{n, 1}}\right) .
\end{gathered}
$$

Having in mind that each $\Lambda_{n, r}$ consists of $2^{n}$ points, we introduce the following parameters:

$$
\begin{aligned}
& m_{1}(\beta)=\lim _{h \rightarrow 0+} \lim _{n \rightarrow \infty} 2^{-n} M_{n}^{(1)}(\beta, h) \\
& m_{2}(\beta)=\lim _{n \rightarrow \infty} \sqrt{2^{-2 n} M_{n}^{(2)}(\beta, 0)}
\end{aligned}
$$




$$
\begin{aligned}
& m_{3}(\beta)=\lim _{n \rightarrow \infty} 2^{-n} \bar{M}_{n}(\beta) \\
& m_{4}(\beta)=\lim _{n \rightarrow \infty} \bar{m}_{n}(\beta) \\
& m_{5}(\beta)=\lim _{n \rightarrow \infty} \sqrt{\tilde{m}_{n}^{(2)}(\beta)} .
\end{aligned}
$$

Let also $m_{l}^{\operatorname{tr}}(\beta), l=1,2$ be defined by expressions (2.11), (3.1) with $H_{\Lambda_{n, r}}^{\operatorname{tr}}(\sigma)$ instead of $H_{\Lambda_{n, r}}(\sigma)$. In fact, only $m_{1}(\beta)$ and $m_{1}^{\operatorname{tr}}(\beta)$ are order parameters in the hierarchical and translation invariant models respectively. Other parameters introduced above are used to prove the positivity of the order parameters for the given values of $\beta$. In what follows, Propositions 2.2, 2.3 and the Griffiths inequality, which hold for all types of the measures $\chi$ considered in this work, imply

$$
m_{2}(\beta) \leqslant m_{2}^{\operatorname{tr}}(\beta)
$$

On the other hand, in the case of classical (nonquantum) spin models one has [7]

$$
m_{2}(\beta) \leqslant m_{1}(\beta) \quad m_{2}^{\operatorname{tr}}(\beta) \leqslant m_{1}^{\operatorname{tr}}(\beta) .
$$

Using these estimates, F.Dyson proved the existence of a long-range order in both models - hierarchical and translation invariant.

Proposition 3.1 (Dyson's theorem) Let $\lambda \in(0,1)$ and the measure $\chi$ be of the form (2.27) Then there exists $\beta_{*}$ such that for $\beta>\beta_{*}, m_{2}(\beta)>0$.

Applying standard arguments (see, e.g., [19]), one can extend the validity of this assertion also to the single-spin measures of the type of (2.28). Much more information about a long-range order in such models has been obtained by P.Bleher in his works [1], [2] (see also [3]). In particular, one can deduce from these papers the following facts:

Proposition 3.2 Let the single-spin measure $\chi$ be of the form (2.28). Then there exists $\beta_{*}>0$ such that for $\beta>\beta_{*}$

$$
m_{4}(\beta)=m_{5}(\beta):=m(\beta)>0 .
$$

For $\beta \rightarrow \beta_{*}+, m(\beta) \rightarrow 0$ holds in such a way that

$$
\lim _{\beta \rightarrow \beta_{*}+} \frac{\log m(\beta)}{\log \left(\beta-\beta_{*}\right)}:=b>0 .
$$

This property is preserved with the same value of the critical index $b$ for small perturbations of the single-spin measure $\chi$.

Directly from definitions (3.1)-(3.9) we get

$$
m_{3}(\beta) \leqslant m_{4}(\beta), \quad m_{5}(\beta) \leqslant m_{2}(\beta) .
$$


Combining these estimates with (3.10), (3.11) and (3.13), we obtain

$$
\begin{aligned}
& m_{3}(\beta) \leqslant m(\beta) \leqslant m_{2}^{\operatorname{tr}}(\beta) \leqslant m_{1}^{\operatorname{tr}}(\beta), \\
& m_{3}(\beta) \leqslant m(\beta) \leqslant m_{2}(\beta) \leqslant m_{1}(\beta) .
\end{aligned}
$$

For our model, the most convenient parameter for the numerical calculation is

$$
m_{3}^{(n)}(\beta):=2^{-n} \bar{M}_{n}(\beta) .
$$

It may be taken as an approximate (finite size) value of $m_{3}(\beta)$. The fact that the latter parameter is a lower bound for the genuine order parameter shows that it may be used to describe the ordering phase transition in the model considered.

It should be noted here that a substantial description of the ordered phase in such hierarchical models was done in papers [1] and [2] where the asymptotics (for $n \rightarrow \infty$ ) of the "small" partition function (2.22) was obtained. In particular, theorem A of paper [1] and theorem 2.1 of paper [2] imply the following assertion:

Proposition 3.3 For any $\lambda \in(0,1)$ there exist positive $\beta_{*}$, the function $\phi(\beta)$, and a sequence of functions $\left\{m_{n}(\beta), n \in \mathbf{N}\right\}$, such that

$$
\begin{aligned}
& \lim _{n \rightarrow \infty} m_{n}(\beta)=m(\beta) ; m_{n}(\beta)>0, \quad \forall \beta>\beta_{*}, \\
& \lim _{\beta \rightarrow \beta_{*}+} \phi(\beta)=+\infty, \quad \phi(\beta)>0, \quad \forall \beta>\beta_{*},
\end{aligned}
$$

where $m(\beta)$ was introduced in (3.12). For these functions and for the density (2.21), the following convergence takes place:

$$
\lim _{n \rightarrow \infty} \sup _{\xi \in \mathbf{R}}\left|T_{n}^{\beta, 0}\left(2^{n / 2} \xi\right)-B\left(\xi ; 2^{n / 2} m_{n}(\beta), \phi(\beta)\right)\right|=0
$$

where

$$
\begin{aligned}
B(\xi ; m, \phi) & =\frac{1}{2}(G(\xi+m ; \phi)+G(\xi-m ; \phi)), \\
G(\xi ; \phi) & =(2 \pi \phi)^{-1 / 2} \exp \left(-\frac{\xi^{2}}{2 \phi}\right) .
\end{aligned}
$$

Let $a_{n}(\beta)$ be defined as $a_{\chi}$ by $(2.33)$ with $\chi=P_{\Lambda_{n, 1}}^{\beta, 0}$. Then the assertion formulated above implies

$$
\lim _{n \rightarrow \infty} a_{n}=2, \quad \forall \beta>\beta_{*} .
$$

Now let us describe the critical point behaviour of the hierarchical model. One can deduce from (3.7) and (3.14)

$$
M_{n}^{(2)}(\beta, 0)=O\left(2^{2 n}\right), \text { for } \beta>\beta_{*} .
$$

On the other hand, for the disordered phase only bulk divergences should appear

$$
M_{n}^{(2)}(\beta, 0)=O\left(2^{n}\right), \text { for } \beta<\beta_{*} .
$$


As for the critical point itself, an intermediate type of divergences is expected

$$
M_{n}^{(2)}\left(\beta_{*}, 0\right)=O\left(2^{n(1+\rho)}\right),
$$

with some $\rho \in(0,1)$. In order to prove this conjecture and to find $\rho$, we proceed as follows. Define the sequence of random variables $\left\{\tau_{n}^{\rho}, n \in \mathbf{N}\right\}$, where

$$
\tau_{n}^{\rho}=2^{-\frac{n}{2}(1+\rho)} \sigma\left(\Lambda_{n, r}\right), \quad \rho \geqslant 0
$$

The probability distribution of each $\tau_{n}^{\rho}$ can be described by means of the measure (2.18), where we put $h=0$. If the sequence $\left\{\tau_{n}^{0}\right\}$ is asymptotically normal, the dependence between the spins is weak or absent as in the case described by the standard central limit theorem. It corresponds to the asymptotics (3.20). In the case of positive $\rho$ in (3.22), one has abnormal normalization of the sums $\sigma(\Lambda)$. For weakly dependent spins, the sequence $\left\{\tau_{n}^{0}\right\}$ is asymptotically normal, then the sequences $\left\{\tau_{n}^{\rho}, \rho>0\right\}$ are asymptotically degenerate at zero. Therefore, the convergence of such a sequence to some nondegenerate random variable would correspond to the appearance of a strong dependence between the spins. The latter is assumed to occur at the critical point of the model. Such critical point convergences were proven.

Proposition 3.4 ( [11] ) Let $\lambda \in\left(0, \frac{1}{2}\right)$ and the measure $\chi$ possess the Lee-Yang property. Then there exists $\beta_{*}>0$ such that: (i) for $\beta=\beta_{*}$, the sequence $\left\{\tau_{n}^{\lambda}\right\}$ is asymptotically normal; (ii) for $\beta<\beta_{*}$ the sequence $\left\{\tau_{n}^{0}\right\}$ is asymptotically normal,

Proposition 3.5 ( [4] ) Let the initial measure $\chi$ be chosen of the form (2.28) with $u=1$. Then there exist $\varepsilon>0, v_{0}>0$, and $\beta_{*}$ such that for $\lambda \in\left(\frac{1}{2}, \frac{1}{2}+\varepsilon\right)$, $v \in\left(0, v_{0}\right), \beta=\beta_{*}$, the sequence $\left\{\tau_{n}^{\lambda}\right\}$ converges to some non-Gaussian random variable.

For some Borel subset $A \subset \mathbf{R}$, we denote

$$
\tilde{P}_{n}^{\beta}(A)=\operatorname{Prob}\left(\tau_{n}^{\lambda} \in A\right) .
$$

In the case where the initial measure $\chi$ is absolutely continuous with respect to the Lebesgue measure on $\mathbf{R}$, these are all $\tilde{P}_{n}^{\beta}$. Let us denote

$$
\frac{\delta \tilde{P}_{n}^{\beta}(\xi)}{\delta \xi}=\tilde{T}_{n}(\xi)
$$

Making use of definition (3.22), one obtains from the recursion relations (2.19), $(2.20)$

$$
\tilde{T}_{n}(\xi) \frac{1}{\tilde{K}_{n}} \exp \left(\beta q \xi^{2}\right) \int_{\mathbf{R}} \tilde{T}_{n-1}\left(2^{-\frac{1-\lambda}{2}} \xi+\eta\right) \tilde{T}_{n-1}\left(2^{-\frac{1-\lambda}{2}} \xi-\eta\right) \mathrm{d} \eta
$$

where

$$
\tilde{K}_{n}=\int_{\mathbf{R}^{2}} \exp \left(\beta q \xi^{2}\right) \tilde{T}_{n-1}\left(2^{-\frac{1-\lambda}{2}} \xi+\eta\right) \tilde{T}_{n-1}\left(2^{-\frac{1-\lambda}{2}} \xi-\eta\right) \mathrm{d} \xi \mathrm{d} \eta
$$


and

$$
q=\frac{1}{2}\left(1-2^{-1-\lambda}\right) J
$$

Thus, a direct corollary of Propositions 3.4 , 3.5 is the following assertion:

Proposition 3.6 (i) Let the conditions of Proposition 3.4 be satisfied. Then there exists $\beta_{*}$ such that for $\beta=\beta_{*}$, the sequence $\left\{\tilde{P}_{n}^{\beta}, n \in \mathbf{N}\right\}$ weakly converges to some Gaussian measure.

(ii) Let the conditions of Proposition 3.5 be satisfied. Then there exists $\beta_{*}$ such that for $\beta=\beta_{*}$, the sequence $\left\{\tilde{P}_{n}^{\beta}, n \in \mathbf{N}\right\}$ weakly converges to some non-Gaussian measure.

(iii) In both cases described above the following asymptotics holds:

$$
M_{n}^{(2)}\left(\beta_{*}, 0\right)=O\left(2^{n(1+\lambda)}\right)
$$

Remark 3.1 The restriction for $\lambda$ which is in the small interval $\left(\frac{1}{2}, \frac{1}{2}+\varepsilon\right)$ mentioned in Proposition 3.5 seems to be purely technical. One can expect that the convergence stated there holds for all $\lambda \in\left(\frac{1}{2}, 1\right)$. It is confirmed by the numerical results given in paper [4]

Hence, having in mind (3.18), one can deduce

Proposition 3.7 Let $\lambda$ be chosen in the interval $(1 / 2,1)$ and $a_{n}(\beta)$ be as in (3.18), then

$$
\begin{aligned}
& \lim _{n \rightarrow \infty} a_{n}(\beta)=0, \quad \text { for } \beta<\beta_{*} ; \\
& \lim _{n \rightarrow \infty} a_{n}\left(\beta_{*}\right)=a \in(0,2) ; \\
& \lim _{n \rightarrow \infty} a_{n}(\beta)=2, \quad \text { for } \beta>\beta_{*} .
\end{aligned}
$$

Now let us consider some consequences of the critical point asymptotics (3.28) just established. First we find how the correlation length $\xi_{n}$, which describes the decay of the spin-spin correlations in $\Lambda_{n .1}$ in such a model, diverges when $n \rightarrow \infty$ and $\beta=\beta_{*}$. It may be used to check the assumptions of the finite size scaling method [8], [9]. We follow the latter paper where the main assumption of this method is formulated as a rule for thermodynamic quantity $A_{L}(t)$, calculated on a finite lattice of linear size $L$, to depend on this size and on $t=\left(\beta_{*}-\beta\right) / \beta_{*}$ as

$$
A_{L}(t)=L^{\rho / \nu} f_{A}(s(L, t)), \quad s(L, t)=L / \xi(t) .
$$

Here a power-law critical singularity for the bulk (i.e. obtained in the thermodynamic limit $L \rightarrow \infty)$ quantity $A=O\left(t^{-\rho}\right)$ is assumed, and $\xi(t)=O\left(t^{-\nu}\right)$ stands for the bulk value of $\xi$. Let us choose $A=\xi$, which means $\rho=\nu$, and put $\beta=\beta_{*}$, that is $t=0$. Then one has in (3.30) $s(L, 0)=0$ which yields in its turn

$$
\xi_{L}(0)=L f_{\xi}(0)
$$


To check this assumption we use definition (3.1) and obtain

$$
M_{n}^{(2)}(\beta, 0)=\sum_{s, s^{\prime} \in \Lambda_{n, r}}<\sigma_{s} \sigma_{s^{\prime}}>_{\Lambda_{n, r}}
$$

The correlation function $<\sigma_{s} \sigma_{s^{\prime}}>_{\Lambda_{n, r}}$ depends on the hierarchical distance between $s$ and $s^{\prime}$. We set

$$
<\sigma_{s} \sigma_{s^{\prime}}>_{\Lambda_{n, 1}}=\Phi_{n}\left(\operatorname{dist}\left(\mathrm{s}, \mathrm{s}^{\prime}\right)\right)
$$

It is known (see [3]) that the so-called "small" critical exponent $\eta$ equals zero for one-dimensional and hierarchical models (see also footnote [11] in paper [9]). Then we can substitute in $(3.32) \Phi_{n}(x)$ in its asymptotic form

$$
\Phi_{n}(x)=\Phi_{0} \exp \left(-\kappa_{n} x\right), \kappa_{n}>0,
$$

where $\kappa_{n}$ stands for the inverse correlation length, i.e. $\kappa_{\Lambda_{n, 1}}:=\kappa_{n}=\xi_{n}^{-1}$. In what follows, the $\xi_{n}$ critical point asymptotics prescribed by the finite size scaling assumption (3.31) reads as

$$
\xi_{n}=O\left(2^{n}\right)
$$

To check whether it really holds, we put

$$
\kappa_{n}=\kappa_{0} 2^{-n \rho},
$$

and find the value of $\rho$ which corresponds to the critical point asymptotics of $M_{n}^{(2)}\left(\beta_{*}, 0\right)(3.28)$. Denote

$$
S_{n}(\lambda, \rho)=\sum_{k=0}^{n} 2^{k-n \lambda} \exp \left(-\kappa_{0} 2^{k-n \rho}\right), \kappa_{0}>0 .
$$

Proposition 3.8 Let the correlation function (3.33) have the form (3.34) with $\kappa_{n}$ obeying (3.36). Then

$$
2^{-n(1+\lambda)} M_{n}^{(2)}\left(\beta_{*}, 0\right)=\frac{\Phi_{0}}{2}\left(2^{-n \lambda}+\exp \left(\kappa_{n}\right) S_{n}(\lambda, \rho)\right) .
$$

Proof. One has

$$
\begin{aligned}
M_{n}^{(2)}(\beta, 0) & =\sum_{s, s^{\prime} \in \Lambda_{n, 1}} \Phi_{0} \exp \left\{-\kappa_{n}\left(2^{n\left(s, s^{\prime}\right)}-1\right)\right\} \\
& =\Phi_{0} \exp \left(\kappa_{n}\right) \sum_{k=0}^{n} \Delta_{n}(k) \exp \left(-\kappa_{n} 2^{k}\right)
\end{aligned}
$$

Here $\Delta_{n}(k)$ stands for the number of pairs $\left\{s, s^{\prime}\right\}$ in $\Lambda_{n, 1}$ such that $n\left(s, s^{\prime}\right)=k$ where the latter is given by (2.6). This number depends on the number $\#\left(\Lambda_{n, r}\right)$ of elements in $\Lambda_{n, r}$ and can easily be computed if its following properties are utilized:

$$
\Delta_{n}(0)=\#\left(\Lambda_{n, r}\right)=2^{n}
$$


Analytic and numerical study ...

$$
\begin{gathered}
\Delta_{n}(n)=2 \#\left(\Lambda_{n-1,1}\right) \#\left(\Lambda_{n-1,2}\right)=2 \cdot 2^{2(n-1)} \\
\Delta_{n}(k)=2 \Delta_{n-1}(k), \quad k<n .
\end{gathered}
$$

Thus, we obtain

$$
\Delta_{n}(k)=2^{n+k-1}, k=1,2 \ldots n .
$$

Inserting this into (3.39), we arrive at

$$
M_{n}^{(2)}(\beta, 0)=\Phi_{0} 2^{n-1}+\frac{\Phi_{0}}{2} \exp \left(\kappa_{n}\right) \sum_{k=0}^{n} 2^{n+k} \exp \left(-\kappa_{n} 2^{k}\right)
$$

Now we use (3.36) and (3.37) and obtain (3.38). Q.E.D.

Proposition 3.9 There exist positive bounds $S^{ \pm}$such that

$$
S^{-} \leqslant S_{n}(\lambda, \lambda) \leqslant S^{+}, \quad \forall n \in \mathbf{N}, \quad \forall \lambda \in(0,1) .
$$

Proof. For the given $\lambda$, let $[n \lambda]$ denote an integer part of $n \lambda$ and $\nu(n, \lambda)=n \lambda-[n \lambda]$. Then one has from (3.37)

$$
S_{n}(\lambda, \lambda)=\sum_{k=-[n \lambda]}^{n-[n \lambda]} 2^{k-\nu(n, \lambda)} \exp \left(-\kappa_{0} 2^{k-\nu(n, \lambda)}\right) .
$$

For $\lambda \in(0,1), n-[n \lambda] \rightarrow+\infty$ when $n$ tends to infinity, the number of summands in the right-hand side of (3.44) will increase to infinity.

Let us consider the function

$$
\psi_{k}(\nu)=2^{k-\nu} \exp \left(-\kappa_{0} 2^{k-\nu}\right), \quad k \in \mathbf{Z}, \quad \nu \in[0,1] .
$$

It is easy to show that for $\lambda \in(0,1)$,

$$
\begin{aligned}
& \psi_{k}(1) \leqslant \psi_{k}(\lambda) \leqslant \psi_{k}(0) \text { for } \kappa_{0} 2^{k} \leqslant 1 \\
& \psi_{k}(0) \leqslant \psi_{k}(\lambda) \leqslant \psi_{k}(1) \text { for } \kappa_{0} 2^{k} \geqslant 2
\end{aligned}
$$

The parameter $-\log _{2} \kappa_{0}$ may or may not belong to the set of integer numbers. In the first case we denote this integer number by $k_{0}$ and conclude that the first line of the estimates (3.46) holds for $k \leqslant k_{0}$, whereas the second one holds for $k \geqslant k_{0}+1$. If the mentioned parameter is not an integer, there exists a unique integer number $k$ obeying the estimate

$$
\kappa_{0}^{-1}<2^{k}<2 \kappa_{0}^{-1} .
$$

We denote this number by $k_{0}$ and conclude that the first line of the estimates (3.46) holds for $k<k_{0}$ and the second one holds for $k>k_{0}$. For $k=k_{0}$, one has the following estimates:

$$
\frac{1}{\kappa_{0} e^{2}} \leqslant \psi_{k_{0}}(\lambda) \leqslant \frac{1}{\kappa_{0} e}
$$


Now we are able to construct the bounds $S^{ \pm}$. We put

$$
S^{-}=\min \left\{\psi_{0}(0), \psi_{0}(1),\left(\kappa_{0} e^{2}\right)^{-1}\right\} .
$$

If $-\log _{2} \kappa_{0}:=k_{0}$ belongs to $\mathbf{Z}$, we set

$$
S^{+}=\sum_{-\infty}^{k_{0}} \psi_{k}(0)+\sum_{k_{0}+1}^{+\infty} \psi_{k}(1) .
$$

For noninteger $\log _{2} \kappa_{0}$ we put

$$
S^{+}=\sum_{-\infty}^{k_{0}-1} \psi_{k}(0)+\frac{1}{\kappa_{0} e}+\sum_{k_{0}+1}^{+\infty} \psi_{k}(1),
$$

where $k_{0}$ is the solution of inequalities (3.47). It is clear that all series in (3.50), (3.51) are convergent. Now we rewrite (3.44)

$$
S_{n}(\lambda, \lambda)=\sum_{k=-[n \lambda]}^{n-[n \lambda]} \psi_{k}(\nu(n, \lambda)) .
$$

Making use of the estimates (3.46), (3.48), one can show that (3.43) holds. Q.E.D. Consider also the asymptotics of $S_{n}(1,1)$. One has

$$
\begin{aligned}
S_{n}(1,1) & =\sum_{k=-n}^{0} 2^{k} \exp \left(-\kappa_{0} 2^{k}\right) \\
& \rightarrow \sum_{k=0}^{\infty} 2^{-k} \exp \left(-\kappa_{0} 2^{-k}\right)<\infty .
\end{aligned}
$$

The corollary of (3.43) and (3.52) can be formulated as the following assertion:

Proposition 3.10 Let $S_{n}(\lambda, \rho)$ be defined by (3.37) with $\lambda, \rho \in(0,1]$. Then for $n \rightarrow$ $\infty$ :

$$
\begin{array}{ll}
S_{n}(\lambda, \rho) \rightarrow+\infty, & \text { for } \quad l<\rho, \\
S_{n}(\lambda, \rho) \rightarrow 0, & \text { for } l>\rho .
\end{array}
$$

As a result, one may conclude that the finite scaling assumption (3.31) should be revised.

Proposition 3.11 Let the inverse correlation length $\kappa_{n}$ have the form (3.36) with $\rho \neq \lambda$, then the asymptotics (3.28) does not hold.

The case of $\rho=\lambda$ in (3.36) is more subtle, it needs some modification of the dependence that is probably caused by the hierarchical nature of the model.

The last question which is to be discussed in this section is the Lee-Yang edge singularities at the critical point. The similar discussion for the case of exactly 
soluble models may be found in [18]. Due to Proposition 2.6 all $P_{\Lambda_{n, r}}^{\beta, 0}$ possess the Lee-Yang property and may be characterized by the location of zeros of their Laplace transforms which are nearest to the origin of the imaginary axis. Let $f_{n}(z)$ be the Laplace transform $(2.25)$ of $P_{\Lambda_{n, r}}^{\beta, 0}$, then its derivatives define the moments of this measure. In particular,

$$
M_{n}^{(l)}(\beta, 0)=\left(D^{l} f_{n}\right)(0), \quad l \in \mathbf{N},
$$

where $M_{n}^{(l)}$ are defined by (3.1). Let $z_{1}^{(n)}$ define the location of the nearest zero of $f_{n}$ and $u_{n}^{(2 k)}$ be semiinvariants of $P_{\Lambda_{n, r}, 0}$ defined by (2.29). Then for $z_{1}^{(n)}$, Proposition 2.4 gives the estimate (2.31) with the just mentioned semiinvariants. In the cases described by Proposition 3.5 or by part (ii) of Proposition 3.6, that is in the cases where the critical point convergence of $\left\{\tilde{P}_{n}^{\beta}\right\}$ holds to a non-Gaussian limit, it is expected that $\left|z_{1}^{(n)}\right|$ tends to zero. We describe this phenomenon as follows:

Proposition 3.12 For $z_{1}^{(n)}$, the following critical point asymptotics holds:

$$
\left|z_{1}^{(n)}\right|=O\left(2^{-n(1+\lambda) / 2}\right)
$$

To prove this statement one uses the estimate (2.31), provided the asymptotics of the semiinvariants are known. Here part (ii) of Proposition 3.6 implies

$$
u_{n}^{(2 k)}=O\left(2^{(1+\lambda) k n}\right)
$$

In particular, $u_{n}^{(2)}=M_{n}^{(2)}\left(\beta_{*}, 0\right)$ has the asymptotics (3.28). By means of (3.54) one simply gets the proof.

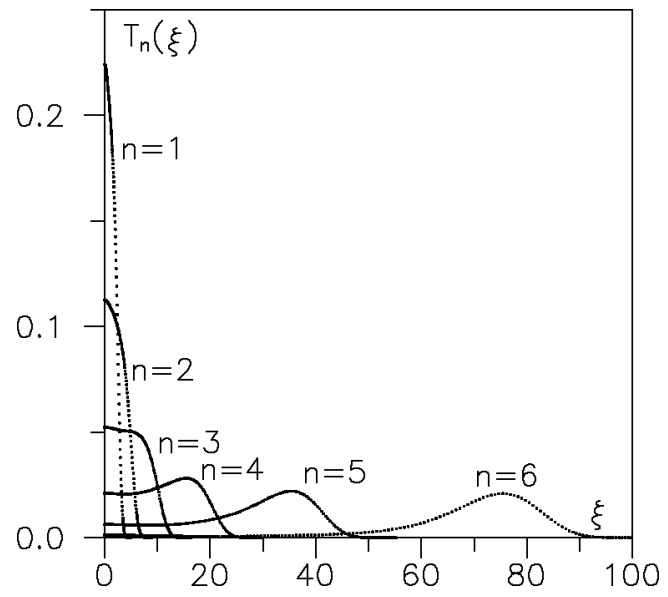

Figure 1. $T_{n}(\xi)$ via $\xi$ for $n=$ $2,3, \ldots, 6$ and $\lambda=2 / 3, \beta^{-1}=0.50$.

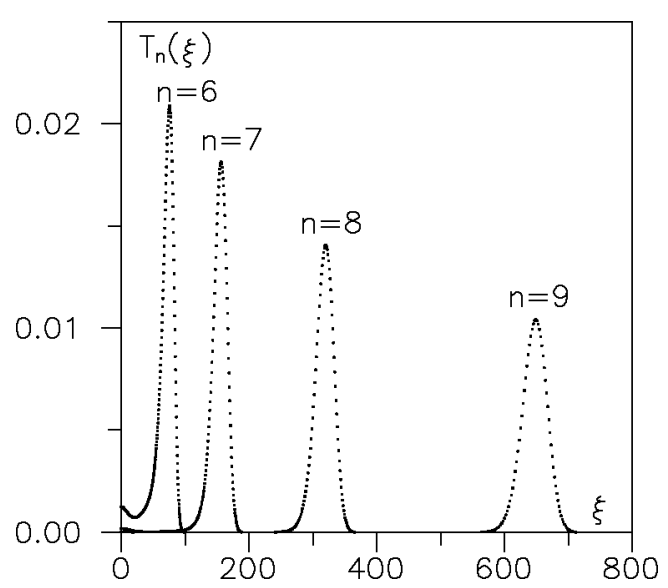

Figure 2. The same as figure 1 for $n=$ $6,7,8,9$. 


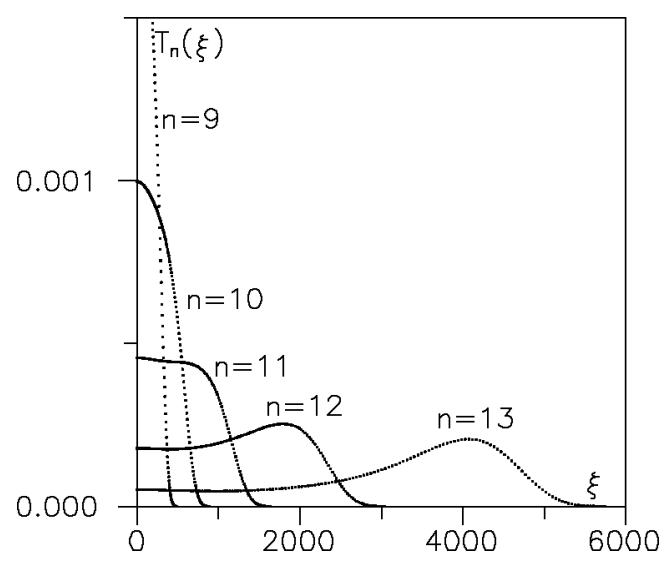

Figure 3. $T_{n}(\xi)$ via $\xi$ for $n=9, \ldots, 13$ and $\lambda=2 / 3, \beta^{-1}=0.61$.

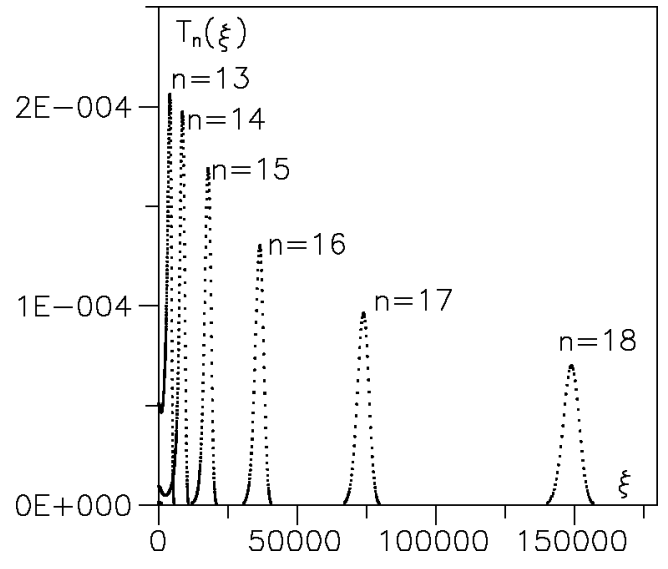

Figure 4. The same as figure 3 for $n=$ $13, \ldots, 18$.

\section{Numerical results}

The main advantage of hierarchical models is that they are very suitable for direct numerical calculations. By means of a relatively weak computer, one can calculate recursively from $(2.23),(2.24)$ the Radon-Nikodym derivatives $T_{n}=T_{n}^{\beta, 0}$, starting from some suitable measure (e.g., of the type of (2.28) ) up to sufficiently high values of $n$. In this section we present the results of such calculations. We choose the initial measure $P_{\Lambda_{0, r}}^{\beta, 0}=\tilde{P}_{0}^{\beta}=\chi$ to be of the form given by (2.28) with $v=1$ and $u=0.1$. This is the simplest non-Gaussian measure possessing the Lee-Yang property. Another choice of $v$ would change only the scale of $\beta$. The dependence of the results on the choice of $u$ will be considered in a separate work. We expect it to have only a quantitative character. The scale of $\beta$ is also defined by the choice of $J$ in (3.27). We have put

$$
J=2 \frac{1-2^{-\lambda}}{1-2^{-1-\lambda}}
$$

that gives

$$
q=1-2^{-\lambda} .
$$

The first object of our investigation is the Radon-Nikodym derivative $T_{n}(\xi)$ defined recursively by $(2.23),(2.24)$ with $T_{0}(\xi)=\mathrm{d} \chi(\xi) / \mathrm{d} \xi$. For $u=0.1$ this function has only one maximum at $\xi=0$. For $\beta=0$, all the $T_{n}$ will have this property. Therefore, for small $\beta$, one may expect this property of $T_{n}$ to hold. On the other hand, one may connect the appearance of a long-range order with the appearance - at some $n_{0}$ - of the maxima of $T_{n_{0}}$ at $\xi= \pm y_{n_{0}}$. The number $n_{0}$ must tend to infinity with $\beta$ approaching $\beta_{*}$ from above. Such a picture was proven by means of the large deviation method [1], [2]. We have obtained it numerically. For the value of the parameter $\lambda$, we have often chosen $2 / 3$ which corresponds to $d=3$ 


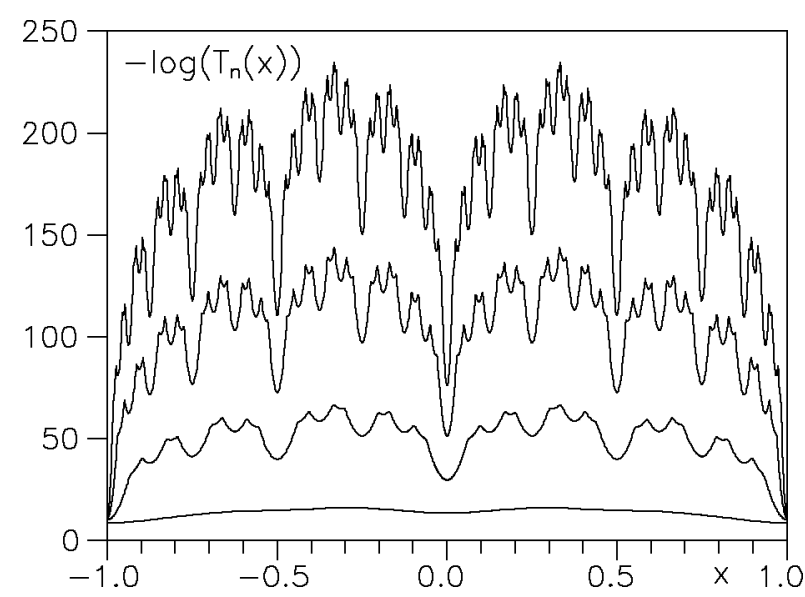

Figure 5. $-\log T_{n}(x)$ via $x=\xi / m_{n}(\beta)$ subsequently for $n=15,20,23,25$, the lowest curve is for $n=15$. if the hierarchical model is used to describe the Yukhnovskii approach to the $d$-dimensional Ising model. Figure 1 shows the evolution of $T_{n}(\xi)$ with $n$ for $\lambda=2 / 3$ and $\beta^{-1}=0.50$. Here $n_{0}=4$. Figure 2 shows further evolution of $T_{n}$ with the same values of the parameters. It can be seen that the new maximum transforms subsequently into a sharp peak, which shows that the corresponding $P_{n}$ becomes more and more close to the distribution concentrated at two points $\pm m_{n}(\beta)$ in full agreement with Proposition 3.3.

Figures 3 and 4 show the same behaviour of $T_{n}$ for $\lambda=2 / 3$ and $\beta^{-1}=0.61$. This behaviour is similar to that of the previous case, but here $n_{0}=12$. Therefore, these two values of $\beta$ are greater than $\beta_{*}$. The behaviour of $T_{n}$ between the peaks $\pm m_{n}(\beta)$ is shown in figure 5. Here the dependence of $-\log T_{n}$ on $x:=\xi / m_{n}(\beta)$ is plotted for $\lambda=0.65, \beta^{-1}=0.624$, and subsequently $n=15,20,23,25$ (the highest curve corresponds to $n=25$ ). This picture was theoretically predicted by P.Bleher in his research [1].

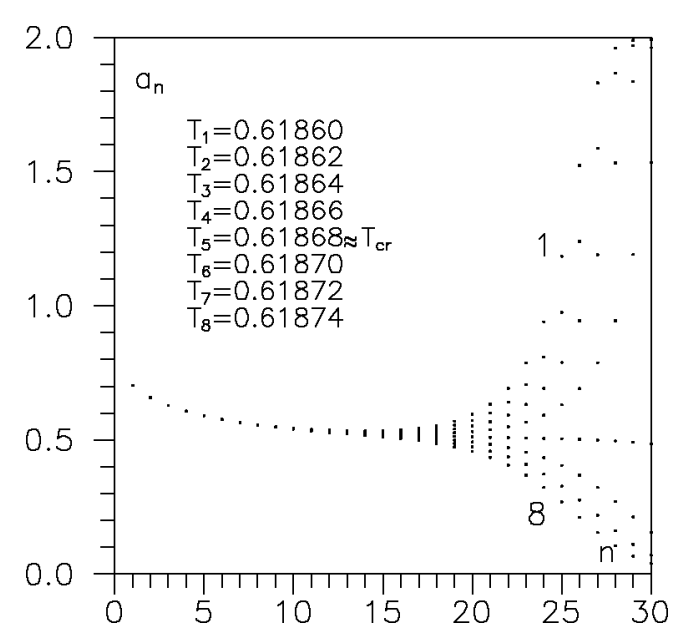

Figure 6. Evolution of $a_{n}(\beta)$ with $n$ for eight subsequent values of $T=\beta^{-1}$.
To calculate the values of the critical temperature $T_{\mathrm{c}}=\beta_{*}^{-1}$ for $\lambda \in\left(\frac{1}{2}, 1\right)$, we use the parameter $a_{n}(\beta)$ described by Proposition 3.7. Hence, we study the evolution of $a_{n}(\beta)$ with $n$ for different fixed values of $\beta$, and $\lambda \in\left(\frac{1}{2}, 1\right)$. Figure 6 shows such an evolution for $\lambda=2 / 3$ and eight values of $T=\beta^{-1}$. It is as described by (3.29), and one may evaluate $T_{\mathrm{cr}}=\beta_{*}^{-1}=0.61868$.

The next object of our investigation is the order parameter. In fact, it is very hard to study the genuine order parameter given by (3.5) directly. Instead of this, we have studied the lowest one, $m_{3}(\beta)$ (see $(3.7),(3.14)$ ). Figure 7 shows the $T:=\beta^{-1}$-dependence of this order parameter for $\lambda=2 / 3$ and three values of $n=10 ; 15 ; 20$. Having this dependence, one can compute the value of the critical index $b$, given by (3.13).

Figure 8 shows the dependence of $b$ and $T_{\text {cr }}$ evaluated from (3.13) on the values of $\lambda$ from the interval $\left(\frac{1}{2}, 1\right)$. 


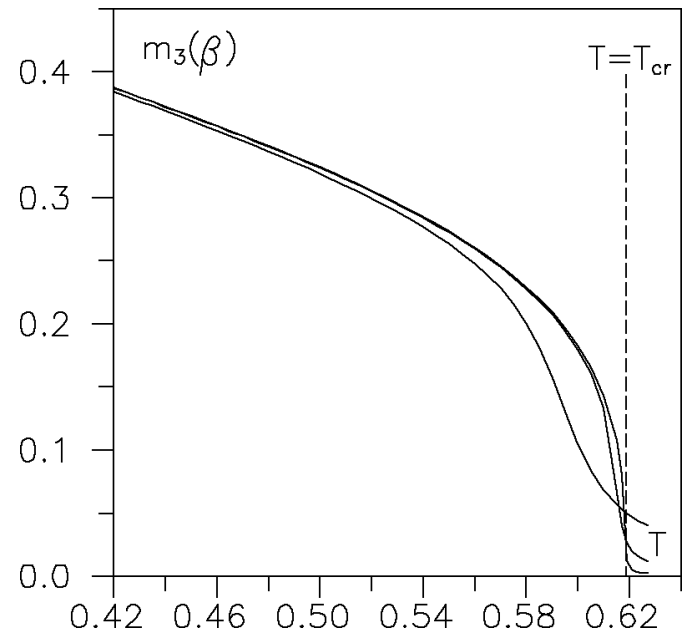

Figure 7. $m_{3}^{(n)}(\beta)$ given by (3.15) via $T=\beta^{-1}$ for $\lambda=2 / 3$ and subsequently $n=10,15,20$, the lowest curve is for $n=10$.

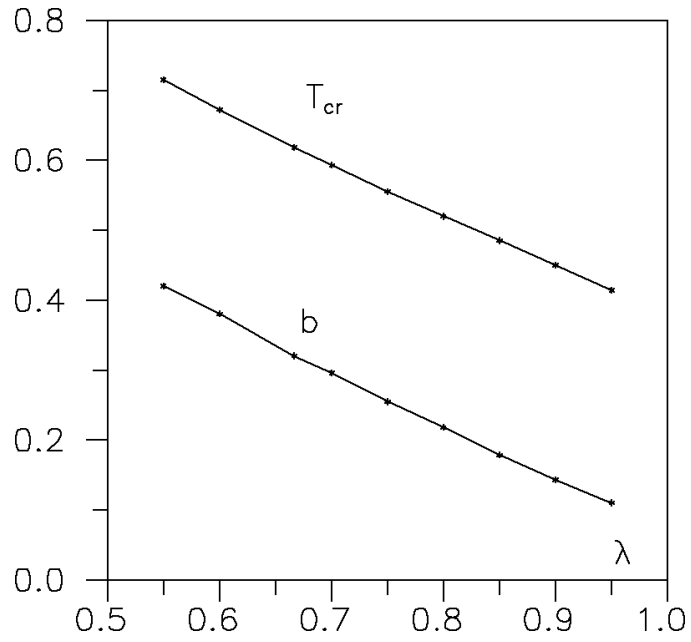

Figure 8. Critical index $b$ defined by $m_{3}(\beta)=A\left(T_{\mathrm{cr}}-T / T_{\mathrm{cr}}\right)^{b}$ and $T_{\mathrm{cr}}$ via $\lambda$.

\section{Acknowledgements}

This work was supported in part by the Fundamental Research Fund of Ukraine (Grant No. 2.4/173).

\section{References}

1. Bleher P.M. Phases separation phenomenon in $\phi^{4}$-hierarchical model. // Teor. Mat. Fiz., 1984, vol. 61, p. 226-240 (in Russian).

2. Bleher P.M. Large deviations theorem near the critical point of $\phi^{4}$-hierarchical model. // Teor. Veroyatnost. i Primenen., 1985, vol. 30, p. 499-510 (in Russian).

3. Bleher P.M., Major P. Critical phenomena and universal exponents in statistical physics. On Dyson's hierarchical model. // Ann. Probability, 1987, vol. 15, p. 431-477.

4. Bleher P.M., Sinai Ja.G. Critical indices for Dyson's asymptotically hierarchical model. // Commun. Math. Phys., 1975, vol. 45, p. 247-278.

5. Dukhovii V.V., Kozlovskii M.P., Pylyuk I.V. Calculation on the microscopic level of the equation of state of 3D Ising model. // Teor. Mat. Fiz., 1996, vol. 107, p. 288-306 (in Russian).

6. Dyson F.J. Existence of a phase transition in a one-dimensional Ising ferromagnets. // Commun. Math. Phys., 1969, vol. 12, p. 91-107.

7. Dyson F.J., Lieb E.H., Simon B. Phase transitions in quantum spin systems with isotropic and nonisotropic interactions. // Journal of Statistical Physics, 1978, vol. 18, p. $335-383$.

8. Fisher M.E. The theory of critical point singularities. In: Stability and Phase Transitions. Moskow, Mir, 1973, p. 245-369 (in Russian). 
9. Jae-Kwon Kim, Adauto J.F. de Souza, D.P.Landau. Numerical computation of finite size scaling functions: An alternative approach to finite size scaling. // Phys. Rev. E, 1996, vol. 54, p. 2291-2297.

10. Kozitsky Yu.V. Hierarchical model of a vector ferromagnet. Self-similar block-spin distributions and the Lee-Yang theorem. // Reports on Math. Phys., 1988, vol. 26, p. 429-445.

11. Kozitsky Yu.V. Hierarchical ferromagnetic vector spin model possessing the Lee-Yang property. Thermodynamic limit at the critical point and above. // Journal of Statistical Physics, 1997, vol. 87, p. 799-820.

12. Kozitsky Yu.V., Melnik N.O. Random vectors with the Lee-Yang property. // Teor. Mat. Fiz., 1989, vol. 78, p. 177-186 (in Russian).

13. Kozitsky Yu.V., Yukhnovskii I.R. Generalized hierarchical model of a scalar ferromagnet in the collective variables method. // Teor. Mat. Fiz., 1982, vol. 51, p. 268-277 (in Russian).

14. Kozlovskii M.P., Korinevskii M.A., Kozitsky Yu.V. An application of the collective variables method in the theory of second order phase transitions. Ising model, cluster models, hierarchical models. In: Problems of Modern Statistical Physics (edited by N.N. Bogolyubov). Kiev, Naukova Dumka, 1985, p. 140-158 (in Russian).

15. Kozlovskii M.P., Pylyuk I.V. Entropy and specific heat of the 3D Ising model as functions of temperature and microscopic parameters of the system. // Phys. Stat. Sol. (b), 1994, vol. 183, p. 243-249.

16. Kozlovskii M.P., Pylyuk I.V., Dukhovii V.V. Equation of state of the 3D Ising model with an exponentially decreasing potential in the external field. // JMMM, 1997, vol. 169 , p. 335-342.

17. Kozlovskii M.P., Pylyuk I.V., Yukhnovskii I.R. Thermodynamic functions of 3D Ising model near the phase transition point with allowance for correction to scaling. I. The case $T>T_{\mathrm{c}}$. // Teor. Mat. Fiz., 1991, vol. 87, p. 293-316; II. The case $T<T_{\mathrm{c}}$. ibid 1991, vol. 87, p. 434-455 (in Russian).

18. Kurtze D.A. The Lee-Yang edge singularity in one-dimensional Ising and N-vector models. // Journal of Statistical Physics., 1993, vol. 30, p. 15-35.

19. Simon B., Griffiths R.B. The $\left(\phi^{4}\right)_{2}$ field theory as a classical Ising model. // Commun. Math. Phys., 1973, vol. 33, p. 145-164.

20. Sinai Ja.G. Self-similar probability distributions. // Teor. Veroyatnost. i Primenen., 1976, vol. 21, p. 63-80 (in Russian).

21. Yukhnovskii I.R. Phase Transitions of the Second Order. The Collective Variables Method. World Scientific Publ. Co., Singapore 1987.

22. Yukhnovskii I.R., Kozlovskii M.P. Description of the phase transition in 3D systems. // JMMM, 1995, vol. 140-144, p. 1477-1478. 


\section{Аналітичне й чисельне дослідження ієрархічної спінової моделі}

\section{Ю.Козицький ${ }^{1,2}$, М.Козловський ${ }^{2}$, Т.Крохмальський ${ }^{2}$}

1 Університет ім. М. Кюрі-Склодовської, Інститут математики, 20-031 Люблін, Польща

2 Інститут фізики конденсованих систем НАН України, 290011 Львів, вул. Свєнціцького, 1

Отримано 7 жовтня 1997 р.

На основі аналітичних і чисельних методів вивчається проста ієрархічна скалярна спінова модель в околі її критичної точки. Аналітично описані залежності сприйнятливості і локалізації нулів статистичної суми моделі скінченого розміру (тобто, великого, але скінченого числа спінів) від кількості спінів поблизу критичної точки. Шляхом аналітичного розрахунку, зокрема, показано, що кореляційна довжина моделі скінченого розміру розбігається в критичній точці слабше ніж це передбачалось скейлінговою теорією. Обчислена критична температура і знайдений критичний показник параметра порядку для різних значень параметра взаємодії.

Ключові слова: ієрархічні моделі, ізінгоподібні моделі, одновимірні системи, критична точка, фазовий перехід

PACS: $05.50 .+q, 64.60 . \mathrm{Cn}, 64.60 . \mathrm{Fr}, 75.10 . \mathrm{Hr}$ 